21st Particles and Nuclei International Conference (PANIC 2017)

International Journal of Modern Physics: Conference Series

Vol. 46 (2018) 1860084 (5 pages)

(C) The Author(s)

DOI: $10.1142 / \mathrm{S} 2010194518600844$

\title{
Masses of Charmed and Bottom Tetraquarks in the Non-Relativistic Quark Model
}

\author{
Zahra Ghalenovi \\ Department of Physics, Kosar University of Bojnord, Bojnord, Iran
}

Published 3 May 2018

\begin{abstract}
Heavy tetraquark states are studied within the diquark-antidiquark picture in the framework of a simple constituent quark model. Considering hyperfine spin and isospin interactions, we predict the masses of the scalar diquarks and of the open and hidden charmed and bottom scalar tetraquarks. Our results indicate the scalar resonances $D_{0}^{*}(2400)$ and $D_{s}(2632)$ have a sizable tetraquark amount in their wave function, while it turns out the scalar state $D_{s 0}^{*}(2317)$ should not be considered as being predominately diquarkantidiquark bound states.
\end{abstract}

Keywords: Scalar tetraquarks; diquark-antidiquark picture; potential model; nonrelativistic limit.

\section{Introduction}

The masses of heavy tetraquarks as diquark-antidiquark bound states were studied in the presence of spin-spin interactions in Ref. [1,2] and later in the comprehensive study of Ref. [3]. In this paper we calculate masses of (hidden and open) charmed and bottom ground-state scalar tetraquarks using a potential model in the non-relativistic limit. As a four-body system, a tetraquark state is quite different from a conventional $q \bar{q}$ meson. We solve the problem in a two-step procedure: first, we use a quark-quark interaction Hamiltonian in order to obtain the mass of a constituent 'good diquark' of the type $\left[q, q^{\prime}\right]$. Second, we regard the diquarks as point-like objects and use a diquark-antidiquark interaction Hamiltonian in order to obtain the tetraquark masses. In both steps we solve the two-body Schrödinger equation by using an Ansatz method. In Sec. 2 we introduce our potential model and present a method to solve the Schrödinger equation in the presence of hyperfine interactions. Our predictions for diquarks and scalar tetraquark masses are presented and discussed in Sec. 3. Finally, a summary and discussion are presented in Sec. 4.

This is an Open Access article published by World Scientific Publishing Company. It is distributed under the terms of the Creative Commons Attribution 4.0 (CC-BY) License. Further distribution of this work is permitted, provided the original work is properly cited. 


\section{Z. Ghalenovi}

\section{The model}

The interaction Hamiltonian for the quark-quark interaction leading to the formation of diquarks is given by

$$
H^{q q}(x)=V^{q q}(x)+H_{h y p}^{q q},
$$

where the potential $V^{q q}(x)$ consists of three parts:

$$
V^{q q}(x)=a x^{2}-\frac{\tau}{x}-C .
$$

The first term $a x^{2}$ is a confining potential and the second term $-\tau / x$ is a Coulomblike potential due to one-gluon exchange processes. $a, \tau$ and $C$ are positive constants. The variable $x$ is the relative quark-quark coordinate. The quantity $H_{h y p}^{q q}$ is the hyperfine interaction given by:

$$
H_{\text {hyp }}(x)=H_{S}(x)+H_{I}(x)+H_{S I}(x),
$$

where $H_{S}(x), H_{I}(x)$, and $H_{S I}(x)$ are spin-spin, isospin-isospin, and spin-isospin interactions, respectively. They read explicitly [4-8]:

$$
\begin{gathered}
H_{S}=A_{S}\left(\frac{1}{\sqrt{\pi} \sigma_{s}}\right)^{3} \exp \left(-\frac{x^{2}}{\sigma_{S}^{2}}\right)\left(\vec{s}_{1} \cdot \vec{s}_{2}\right), \\
H_{I}=A_{I}\left(\frac{1}{\sqrt{\pi} \sigma_{I}}\right)^{3} \exp \left(-\frac{x^{2}}{\sigma_{I}^{2}}\right)\left(\vec{t}_{1} \cdot \vec{t}_{2}\right), \\
H_{S I}=A_{S I}\left(\frac{1}{\sqrt{\pi} \sigma_{S I}}\right)^{3} \exp \left(-\frac{x^{2}}{\sigma_{S I}^{2}}\right)\left(\vec{s}_{1} \cdot \vec{s}_{2}\right)\left(\vec{t}_{1} \cdot \vec{t}_{2}\right),
\end{gathered}
$$

where $s_{i}$ and $t_{i}$ are the spin and isospin operators of the $i$-th quark, respectively, while $A_{k}$ and $\sigma_{k}$ with $k=S, I, S I$ are constants. Next, we turn to the diquarkantidiquark potential. First, we recall that the one-gluon exchange potential is such that the quark-antiquark potential and quark-quark potentials are related by $V_{q \bar{q}}=2 V_{q q}$. When turning to the interaction between a good diquark and a good antidiquark, we assume the same form as for a quark-antiquark pair [9]. Thus, taking into account the factor 2 , we get for a diquark-antidiquark system:

$$
H^{D \bar{D}}(x)=V^{D \bar{D}}(x)+H_{\text {hyp }}^{D \bar{D}},
$$

where the potential $V^{D \bar{D}}(x)$ reads

$$
V^{D \bar{D}}(x)=2 a x^{2}-\frac{2 \tau}{x}-C
$$

The variable $x$ is now the relative diquark-antidiquark coordinate and $H_{h y p}^{D \bar{D}}$ has the same form as $H_{\text {hyp }}$ in Eq. (3).

Since the potential (2) is assumed to depend on $x$ only, one can factor out the angular part of the two-body wave function. The remaining radial part of the wave 
function for the two-body problem with the unperturbed potential $V^{q q}(x)$ is then determined by the Schrödinger equation

$$
\left[\frac{d^{2}}{d x^{2}}+\frac{2}{x} \frac{d}{d x}-\frac{l(l+1)}{x^{2}}\right] \psi_{l}(x)=-2 m\left[E_{l}-V^{q q}(x)\right] \psi_{l}(x),
$$

where $\psi_{l}(x)$ is the radial wave function, $l$ is the angular quantum number, and $m$ is the reduced mass of the two-body system containing the constituent quarks (and, subsequently, diquarks). In order to solve Eq. (9) we assume the following transformation for $l=0$ (the ground state) $[5,10]$

$$
\psi(x)=x^{-1} \exp \left(-\frac{1}{2} \alpha x^{2}+\delta \ln x\right),
$$

where $\alpha$ and $\delta$ are constants. By substituting Eq. (10) in Eq. (9) we can get $\alpha=$ $\sqrt{2 m a}$, and $\delta=1[5,10]$, and regarding $\omega=\sqrt{\frac{2 a}{m}}$ for the oscillating term in Eq. (2), the energy eigenvalue is obtained as follows

$$
E=\frac{3 \omega}{2}-\frac{m \tau^{2}}{2}-C
$$

By determination of $\alpha$ and $\delta$, one can get the eigenfunction as $[5,10]$

$$
\psi=N e^{-\frac{m \omega}{2} x^{2}},
$$

where $N$ is the normalization constant. The very same mathematical problem needs to be solved for the diquark-antidiquark state by treating (anti)diquarks as point particles under the influence of the potential (8). The energy eigenvalue $E_{0, D \bar{D}}$ of the tetraquark ground state $(l=0)$ is then calculated in the same way.

\subsection{Diquarks}

The diquark masses obtain a contribution from the constituent quark masses as well as from the confining and the spin-isospin-dependent interactions:

$$
M_{\text {diquark }}=m_{q 1}+m_{q 2}+E_{0, q q}+\left\langle H_{\text {hyp }}\right\rangle,
$$

where $m_{q i}$ is the mass of $i$-th quark and $E_{0, q q}$ is the ground-state energy calculated in the previous section. The first-order energy correction from the non-confining potential $\left\langle H_{\text {hyp }}\right\rangle$ is calculated using the unperturbed wave function obtained in Secs. 2.2 and 2.3. For the numerical evaluation, we use the light and heavy quark masses and the parameters $a$ and $\tau$ from Ref. [4], while the hyperfine potential parameters are taken from Refs. $[5,8]$. The obtained masses of scalar diquark are shown in Table 1 , and compared with the ones of other theoretical works $[3,11,12]$.

\subsection{Heavy scalar tetraquarks}

Once the diquark masses are calculated, we can evaluate the tetraquark masses by following the same steps. The explicit expression reads

$$
M_{\text {tetraquark }}=m_{\text {diquark }}+m_{\text {antidiquark }}+E_{0, D \bar{D}}+\left\langle H_{\text {hyp }}^{D \bar{D}}\right\rangle .
$$


Table 1. Diquark masses (in $\mathrm{MeV}$ ).

\begin{tabular}{cccccc}
\hline Diquark & Our Model & Ref. [4] & Ref. [3] & Ref. [11] & Ref. [12] \\
\hline$[q q]$ & 587 & 527 & 710 & 395 & 441 \\
{$[q s]$} & 828 & 784 & 948 & 590 & 659 \\
{$[q c]$} & 2012 & 2012 & 1973 & 1933 & 1980 \\
{$[s c]$} & 2219 & 2213 & 2091 & - & 2120 \\
{$[q b]$} & 5414 & 5371 & 5359 & - & 5140 \\
{$[s b]$} & 5583 & 5563 & 5462 & - & 5210 \\
\hline
\end{tabular}

Table 2. Masses of open charmed and bottom tetraquarks (in MeV).

\begin{tabular}{ccccccc}
\hline Tetraquark & Our model & Ref. [4] & Ref. [4] & Ref. [3] & Ref. [2] & Exp. [13, 14] \\
\hline$[c q \bar{q} \bar{q}]$ & 2464 & 2398 & 2426 & 2399 & - & $D_{0}^{*}(2400)$ \\
{$[c q \bar{q} \bar{s}]$} & 2629 & 2618 & 2600 & 2619 & 2371 & $D_{s 0}^{*}(2317), D_{s 0}^{*}(2632)$ \\
{$[c s \bar{q} \bar{s}]$} & 2789 & 2855 & 2798 & 2753 & - & - \\
\hline$[b q \bar{q} \bar{q}]$ & 5788 & 5763 & 5748 & 5758 & - & - \\
{$[b q \bar{q} \bar{s}]$} & 5929 & 5980 & 5901 & 5997 & - & - \\
{$[b s \bar{s} \bar{q}]$} & 6085 & 6217 & 6103 & 6108 & - & - \\
\hline
\end{tabular}

Table 3. Masses of double-hidden charmed and bottom scalar tetraquarks(in $\mathrm{MeV}$ ).

\begin{tabular}{cccccc}
\hline Tetraquark & Our model & Ref. [4] & Ref. [4] & Ref. [15,16] & Ref. [2] \\
\hline$[c q \bar{c} \bar{q}]$ & 3625 & 3807 & 3662 & 3812 & 3723 \\
{$[c q \bar{c} \bar{s}]$} & 3768 & 4043 & 3862 & 3922 & - \\
{$[c s \bar{c} \bar{s}]$} & 3920 & 4268 & 4050 & 4051 & - \\
\hline$[b q \bar{b} \bar{q}]$ & 9670 & 10521 & 10044 & 10471 & - \\
{$[b q \bar{b} \bar{s}]$} & 9812 & 10747 & 10228 & 10572 & - \\
{$[b s \bar{b} \bar{s}]$} & 10001 & 10973 & 10412 & 10662 & - \\
\hline
\end{tabular}

The results for open charmed and bottom tetraquarks are listed in Tables 2 and 3 and compared with other theoretical predictions $[2,3]$ and experimental candidates $[13,14]$. The masses of the tetraquarks are indeed similar in all theoretical approaches.

\section{Summary}

In this work we have calculated the masses of the ground-state heavy scalar tetraquarks in the framework of a non-relativistic approach. The results for the scalar diquarks are shown in Table 1, while the heavy scalar tetraquarks are summarized in Table 2 and Table 3. Our results for the masses show that the resonance $D_{s 0}^{*}(2317)$ is too light to be predominantly a tetraquark state of the type $c q \bar{q} \bar{s}$. On the other hand, the state $D_{0}^{*}(2400)$ and the putative $D_{s 0}^{*}(2632)$ can contain 
an important tetraquark component in their flavor wave function $(c q \bar{q} \bar{q}$ and $c q \bar{q} \bar{s}$, respectively).

\section{References}

1. L. Maiani, V. Riquer, F. Piccinini and A. D. Polosa, Phys. Rev. D 72 (2005) 031502.

2. L. Maiani, F. Piccinini, A. D. Polosa and V. Riquer, Phys. Rev. D 71 (2005) 014028.

3. D. Ebert, R. N. Faustov and V. O. Galkin, Phys. Lett. B 696 (2011) 241.

4. Z. Ghalenovi, A. A. Rajabi, S. x. Qin and D. H. Rischke, Mod. Phys. Lett. A 29 (2014) 1450106 .

5. Z. Ghalenovi, A. A. Rajabi and A. Tavakolinezhad, Int. J. Mod. Phys. E 21 (2012) 1250057.

6. Z. Ghalenovi, A. A. Rajabi and M. Hamzavi, Acta Phys. Polon. B 42 (2011) 1849.

7. H. Hassanabadi, A. A. Rajabi and S. Zarrinkamar, Mod. Phys. Lett. A 23 (2008) 527.

8. M. M. Giannini, E. Santopinto and A. Vassallo, Prog. Part. Nucl. Phys. 50 (2003) 263.

9. B. Silvestre-Brac, Few Body Syst. 20, 1 (1996).

10. Z. Ghalenovi and M. Moazzen, Eur. Phys. J. Plus 132, no. 8, 354 (2017).

11. L. Maiani, F. Piccinini, A. D. Polosa and V. Riquer, Phys. Rev. Lett. 93 (2004) 212002.

12. B. Chakrabarti et al., Acta. Phys. Plo. B 411 (2010).

13. K. A. Olive et al. (Particle Data Group), Chin. Phys. C38, 090001 (2014).

14. A. V. Evdokimov et al. [SELEX Collaboration], Phys. Rev. Lett. 93 (2004) 242001.

15. D. Ebert, R. N. Faustov and V. O. Galkin, Phys. Atom. Nucl. 72 (2009) 178.

16. D. Ebert, R. N. Faustov and V. O. Galkin, Eur. Phys. J. C 58 (2008) 399. 